\title{
A MAGNETICALLY GUIDED SLOW POSITRON BEAM FOR DEFECT STUDIES
}

\author{
W. Anwand, H.-R. Kissener and G. Brauer \\ Positron Group of TU Dresden at Research Centre Rossendorf Inc. \\ P.O. Box 510119, D-01314 Dresden, Germany
}

The design and construction of a compact, magnetically guided slow positron beam is discussed. The system uses a $30 \mathrm{mCi}^{22} \mathrm{Na}$ source. It consists of three main parts: (i) the source chamber with a tungsten foil transmission moderator and the extraction optics, (ii) the beam line with magnetic beam guidance, a bent tube for the separation of the fast positrons and the accelerator stage, (iii) the target chamber with the sample holder and the detector electronics. The energy of the incident positrons can be varied from $30 \mathrm{eV}$ up to $50 \mathrm{keV}$. Furthermore source geometries, pre-acceleration, main acceleration sections and various magnetic induction profiles have been considered, such as (i) rectangular, conical and bent Wehnelt electrodes, (ii) pre-accelerator voltage shared over several electrodes, (iii) weak, strong, constant and $z$-dependent $B$-profiles, (iv) geometric options in the main accelerator region, (v) purely electrostatic and combined electric/magnetic fields. The beam is mainly designed for defect profile studies in ultra high vacuum conditions.

PACS numbers: $06.90 .+v, 07.90 .+c$

\section{Introduction}

The aim of our work is to build a relatively simple slow positron system in a short time and without high financial costs. Furthermore, the design and the construction of the Slow POsitroN System Of Rossendorf (SPONSOR) should make it possible for the equipment to be expanded step by step. For this reason we decided that the system should be of magnetic transport type as this provides the simplest means by which a slow positron beam may be transported from its source point to its intended target $[1,2]$. For the separation of the fast positrons we use a bent tube instead of a more complicated $E \times B$ filter.

\section{Design and performance}

The beam line and the magnetic coils are shown in Fig. 1. Only nonmagnetic components are used for the beam sections in order to avoid influences on the magnetic field. The frame made of light-weight concrete blocks connected by aluminium profiles and covered with a thick wooden board was designed to provide good stability. It supports the whole beam construction. 


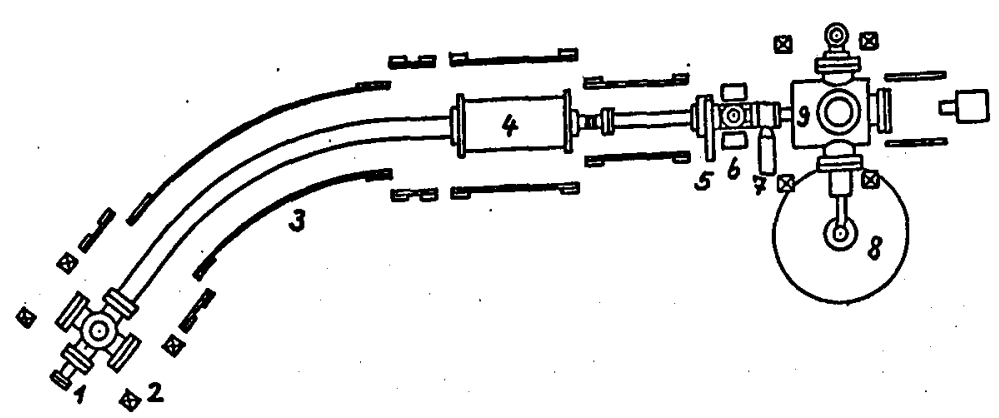

Fig. 1. View of the beam line and the magnetic coil arrangement $(1$ - source, 2 Helmholtz coils, 3 - solenoids, 4 - accelerator, 5 - Iris aperture, 6 - beam movement coils, 7 - valve, 8 - detector, 9 - sample chamber).

Positrons from a $30 \mathrm{mCi}^{22} \mathrm{Na}$ source pass a tungsten moderator foil of about $6 \mathrm{~mm}$ diameter and a thickness of $5 \mu \mathrm{m}$. The positrons are pulled off by a $30 \mathrm{eV}$ beam formation section. To increase the moderation efficiency and to decrease the energy spread of the emitted positrons a heating of the whole moderator foil before its use is necessary [3]. It is planned to make this heat treatment outside of the positron beam system in a special vacuum chamber. The tungsten foil will be fastened between tungsten meshes. The tungsten meshes themselves will be connected with copper holders and copper wires. A current through the tungsten meshes makes possible the heating of the tungsten foil.

The whole beam will be guided from the source to the sample by 4 Helmholtz coils and 8 solenoids. The solenoids are polyethylene cored and wound with $2 \mathrm{~mm}$ diameter insulated copper wire. The same type of wire was used for the Helmholtz coils. These coils are self-supporting without core. The total beam length is about $3 \mathrm{~m}$. The standard option for solid state defect studies adopts an axial magnetic field of $B=100$ Gs through the whole apparatus. By switching off one or two IIelmholtz coils, a softly rising $B$-field over the source can be provided. A bent sector with about $50^{\circ}$ deflection angle and a curvature radius of about $1.2 \mathrm{~m}$ reduces the contamination by fast positrons and other background. For the accelerator we use a section of an ion accelerator.

In the standard option ( $B=100 \mathrm{Gs}$ ) the magnetic force dominates over the electric one by orders of magnitude, except for the main accelerator region. The details of the beam formation and main accelerator geometry are then not critical: the beam spot at the source moves almost unchanged till the entrance to the main accelerator, if the axial magnetic induction is constant up to a few percent.

In order to allow beam compression already in the source region we also considered weaker fields and linearly increasing induction along the beam axis. In these modes, the spot size and the beam divergence before entrance to the bent transport unit sensitively depend on: the size, shape, number, positions and potential settings of the extraction electrodes. To accelerate the positrons up to $50 \mathrm{keV}$ a potential gradient has to be created along the beam line of the positrons. In order to do this we float the entire source part of the beam up to the required 
positive voltage and keep the sample chamber and all solenoids and Helmholtz coils at ground potential. The accelerator forms the break between these potentials and consists of 11 stages. First calculations have shown that the positrons can be additionally focused during their way through the accelerator.

At the end of the accelerator we use an Iris aperture to clip the beam to an useful diameter corresponding to the sample size. The straight connector stage which follows contains a vacuum valve to separate the source and sample chamber parts of the vacuum system. Furthermore, two pairs of magnetic coils are placed at $90^{\circ}$ to the beam line. Their effect is to move the beam in a horizontal and vertical plane to compensate for any movement induced by the accelerator and to keep the beam in the central position. The beam position itself is shown on a phosphor plate behind a channel electron multiplier array (CEMA).

The sample chamber forms the end section of the whole beam. It is a cylinder of $220 \mathrm{~mm}$ diameter and incorporates 6 flanges for positron entry, detection of the gamma rays, the vacuum pump and vacuum gauge, the sample manipulator (including heating), CEMA beam detector and the electrical feedthroughs. Currently the design and the construction of the beam has been completed and most of the system components have been realised. The positron system will be put into operation in the early spring of 1995.

\section{Source region}

Figure 2 shows trajectories for a source geometry with plane source front, spherical Wehnelt deceleration electrode and short tubes sharing about $30 \mathrm{eV}$ pull-out potential for $B=0$ and $B=100$ Gs. The Wehnelt electrode should roughly compensate the mean energy of the moderated electrons. For $B=100 \mathrm{Gs}$ the spot is transferred almost without change of size. For weaker $B$-fields the pre-acceleration unit provides some beam shaping. In a smoothly increasing $B$-field the spot size before entrance to the bent beam section can be reduced below the source diameter. In the limit of vanishing $B$ a smooth curvature of the deceleration electrode allows a better beam shaping than conical or rectangular electrodes. An additional tube with source potential, just after the Wehnelt electrode, extends the
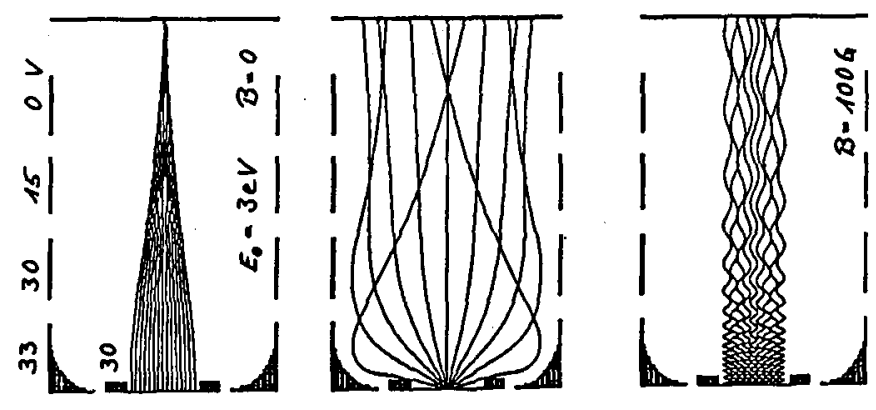

Fig. 2. Trend of trajectories in a source geometry with spherical deceleration electrode. 
efficient beam shaping region. In the standard option with a strong axial magnetic field the influence of the geometric variation is barely visible.

\section{Magnetic field configuration}

The most transparent and least power-consuming guide field is created by a long solenoid tailored to the vacuum system. The simplest laboratory setup is a set of short solenoids (Helmholtz coils), which provide a pulsating magnetic field and cost more electric power. The magnetic configuration of SPONSOR (Fig. 3) is a mixture of long ( $S 1$ to $S 6$ ) and short (H1 to $H 4$ ) solenoids, which create a flat plateau. The bumps in the $B$-profile between neighbouring coils with different

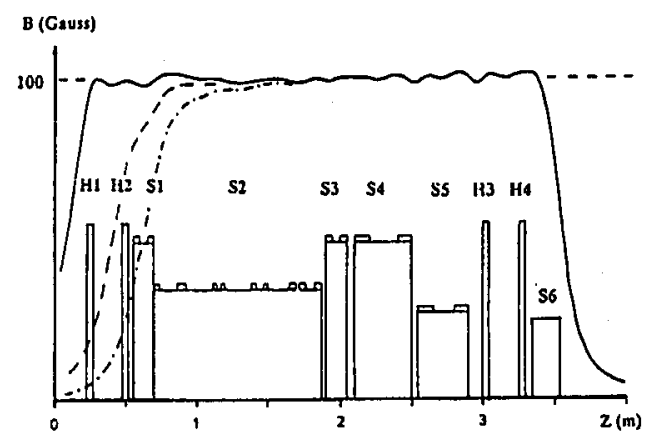

Fig. 3. Calculated $B$-profiles - full curve: standard version, dashed curve: $H 1$ switched off, dash-dotted curve: $H 1$ and $H 2$ switched off.

radii or large spaces are compensated by additional windings on both ends of each solenoid. This makes possible a nearly constant magnetic field of $0.01 \mathrm{~T}$. A more advanced design might involve variable winding densities and/or carrier coil radii. The calculated $B$-profile shown in Fig. 3 has been obtained by fit under constraint of a small number of current supplies. Hence, the flatness might be further improved.

The field regions near the source $(H 1, H 2, S 1)$ and between the exit of the high-potential bent tube section $(S 3)$ and the first accelerator aperture (within $S 4)$ require a careful experimental tuning. The profile $B(z)$ at the accelerator exit and in the drift tube $(S 5)$ is not critical. The solenoid $S 6$ may serve for beam focusing.

\section{Trajectories in the accelerator region}

In the accelerator region the lengths of the Larmor spirals increase proportionally to the instantaneous longitudinal positron momentum. The accelerating field affects also the transversal momentum of the positrons and, hence, their spiral radii. Of particular interest are the fields just at the accelerator entrance where the positron beam is most sensitive.

Figure 4 shows trajectories in the schematized accelerator box. The real unit contains equidistant plane apertures, with the inner radii decreasing linearly in 


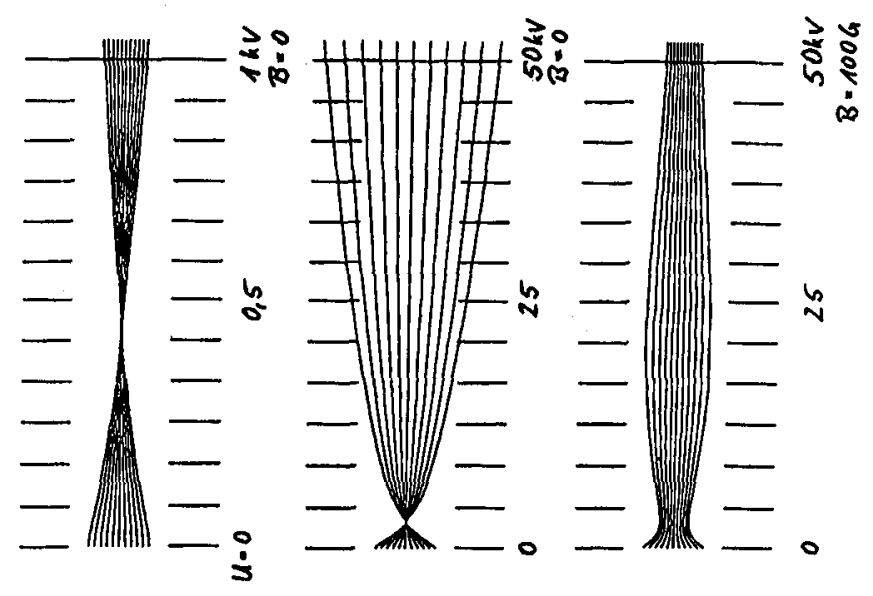

Fig. 4. Trend of the trajectories in the (schematized) accelerator unit (extraction energy $\left.E_{0}=30 \mathrm{~V}\right)$.

the lower half and increasing linearly in the upper half (this shape served for suppressing effects due to secondary electrons in ion accelerator facilities). For $B=0$ the trajectories are notably affected when replacing the double-cone geometry by a single-cone one. The effect is of course energy-dependent. In an axial magnetic field of $100 \mathrm{Gs}$, however, details of the above accelerator geometries influence the trajectories mainly by shadowing.

Earlier studies of purely electrostatic multi-component accelerators have shown that the energy dependence of the cardinal elements of a system with equidistant electrodes can be partly compensated by optimizing the potential settings of the segments: e.g. by reducing of the number of active electrodes (by shunting downstream diaphragms) in the mean-energy regime. In a strong axial magnetic field these strategies lose their efficiency.

\section{Acknowledgments}

Regarding the design of slow positron beams the authors acknowledge useful discussions with W. Triftshäuser, P.G. Coleman, R. Krause-Rehberg and L. Liszkay. The possibility to perform some defect profiling measurements at the magnetically-guided slow positron beam at the UEA Norwich has been especially helpful. We are thankful to R. Krause-Rehberg for the support of his group in the preparation of the ${ }^{22} \mathrm{Na}$ source and to Zs. Kajcsos for the support in the manufacture of some components of the beam.

\section{References}

[1] P.J. Schultz, G.R. Massoumi, P.J. Simpson, Positron Beams for Solids and Surfaces, AIP Conference Proceeding 218, American Institute of Physics, New York 1990.

[2] E. Ottewitte, A.H. Weiss, Slow Positron Techniques for Solids and Surfaces, AIP Conference Proceedings 303, American Institute of Physics, New York 1994.

[3] P. Willutzki, J. Stoermer, D.T. Britton, G. Koegel, P. Sperr, W. Triftshaeuser, in Ref. [2], p. 542. 